\title{
Overlapping Decentralized Controller Design for Descriptor-type Systems with Distributed Time-delay
}

\author{
ALTUĞ İFTAR \\ Department of Electrical and Electronics Engineering \\ Eskişehir Technical University \\ 26555 Eskişehir, TURKEY
}

\begin{abstract}
Decentralized controller design using overlapping decompositions is considered for descriptor-type systems with distributed time-delay. The approach is based on the principle of extension. In this approach, a given large-scale system is decomposed overlappingly into a number of subsystems and expanded such that the overlapping parts appear as disjoint. A decentralized controller is then designed for the expanded system. This controller is then contracted for implementation on the original system. It is shown that if the decentralized controllers are designed to stabilize the expanded system and to achieve certain performance, then the contracted controller, which would have an overlapping decentralized structure, will stabilize the originalsystemandwillachievecorrespondingperformance.
\end{abstract}

Key Words - Time-delay Systems, Descriptor-type Systems, Distributed Time-delay, Decentralized Control, Controller Design, OverlappingDecompositions

Received: March 8, 2021. Revised: August 27, 2021. Accepted: September 15, 2021. Published: September $27,2021$.

\section{Introduction}

Many practical systems may involve time-delays [1]. This is especially true for large-scale systems [2]. Systems which involve time-delays are usually named as time-delay systems [3]. Time-delays in a time-delay system can be discrete or distributed [4]. Systems with distributed time-delay may appear in many applications such as logistics [5], traffic flow [6], [7], [8], combustion [9], neural networks [10], [11], and biology [12], [13]. Furthermore, some systems may involve both discrete and distributed time-delays together [14]. Using Dirac delta functions, however, discrete time-delays may be represented as a distributed time-delay [15]. Thus, in this sense, systems with distributed time-delay are more general than systems involving only discrete time-delays.

Many time-delay systems can be modeled using delaydifferential equations [16]. Delay-differential equations alone, however, may not be sufficient to model some timedelay systems. In some cases, delay-differential equations may need to be coupled with delay-algebraic equations. Such systems are usually named as descriptor-type timedelay systems [17]. Telerobotic systems [18] are one example of descriptor-type time-delay systems. The response of descriptor-type systems may be discontinuous and even impulsive [17]. Thus, dealing with descriptor-type systems is, in general, more challenging.

To analyze or design a controller for a large-scale system may first require to decompose the system into smaller subsystems [19]. However, many large-scale systems may have an overlapping part through which subsystems are interconnected [20]. Disjoint decompositions may not be useful for such systems. It has been shown, however, that the overlapping decompositions approach [21] may produce useful decompositions for such systems (e.g., see [22][30]). Overlapping decompositions approach is based on the principle of inclusion [31]. A special case of inclusion, which is especially useful in controller design is extension, which was first introduced in [32] for finite-dimensional systems. The principle of extension has first been extended to descriptor-type systems with distributed time-delay in [33]. Controller design for such systems was then considered in [34]. However, overlapping decompositions was considered neither in [33] nor in [34].

In the present work, we consider decentralized controller design using overlapping decompositions for linear timeinvariant (LTI) descriptor-type systems with distributed timedelay. We give the necessary background in Sections 2 and 3 , which summarize the results of [33] and [34], respectively. Controller design using overlapping decompositions is then presented in Section 4. Some concluding remarks are finally given in Section 5.

Throughout the paper, $\mathbf{R}$ and $\mathbf{R}_{+}$denote the sets of, respectively, real numbers and non-negative real numbers. For positive integers $k$ and $l, \mathbf{R}^{k}$ and $\mathbf{R}^{k \times l}$ denote the spaces of, respectively, $k$-dimensional real vectors and $k \times l$ dimensional real matrices. For $x \in \mathbf{R}^{k},\|x\|$ is the 2norm of $x$ and, for $X \in \mathbf{R}^{k \times l},\|X\|$ is the induced 2norm (i.e., the maximum singular value) of $X . I_{k}$ denotes the $k \times k$-dimensional identity matrix and 0 may denote a zero matrix of appropriate dimensions or a matrix function 
which is identically zero. Finally, bdiag $(\cdots)$ denotes a block diagonal matrix with indicated blocks on the main diagonal and $\operatorname{rank}(\cdot)$ denotes the rank of the indicated matrix.

For $M_{0} \in \mathbf{R}^{n \times p}$ and $M(\cdot):[-\tau, 0] \rightarrow \mathbf{R}^{n \times r}$, we say that $\left[\begin{array}{cc}M_{0} & M(\cdot)\end{array}\right]$ has full row-rank, if for any $\xi \in \mathbf{R}^{n}$, there exist $u_{0} \in \mathbf{R}^{p}$ and $\varphi:[-\tau, 0] \rightarrow \mathbf{R}^{r}$ such that $\xi=$ $M_{0} u_{0}+\int_{-\tau}^{0} M(\theta) \varphi(\theta) d \theta$.

\section{Extension Pronciple}

In this section, we summarize the results of [33], which were also presented in [34]. Consider two LTI time-delay systems with distributed time-delay, $\Sigma$ :

$$
\begin{gathered}
E_{0} \dot{x}(t)=A_{0} x(t)+B_{0} u(t)+\int_{-\tau}^{0}(A(\theta) x(t+\theta) \\
+B(\theta) u(t+\theta)) d \theta \\
y(t)=C_{0} x(t)+\int_{-\tau}^{0} C(\theta) x(t+\theta) d \theta
\end{gathered}
$$

and $\hat{\Sigma}$ :

$$
\begin{gathered}
\hat{E}_{0} \dot{\hat{x}}(t)=\hat{A}_{0} \hat{x}(t)+\hat{B}_{0} \hat{u}(t)+\int_{-\tau}^{0}(\hat{A}(\theta) \hat{x}(t+\theta) \\
+\hat{B}(\theta) \hat{u}(t+\theta)) d \theta \\
\hat{y}(t)=\hat{C}_{0} \hat{x}(t)+\int_{-\tau}^{0} \hat{C}(\theta) \hat{x}(t+\theta) d \theta
\end{gathered}
$$

where $x(t) \in \mathbf{R}^{n}$ and $\hat{x}(t) \in \mathbf{R}^{\hat{n}}$ are the state, $u(t) \in \mathbf{R}^{p}$ and $\hat{u}(t) \in \mathbf{R}^{\hat{p}}$ are the input, and $y(t) \in \mathbf{R}^{q}$ and $\hat{y}(t) \in \mathbf{R}^{\hat{q}}$ are the output vectors of, respectively, $\Sigma$ and $\hat{\Sigma}$ at time $t$. $\tau>0$ is the maximum time-delay in $\Sigma$ and $\hat{\Sigma}$. It is assumed that the dimensions of the state, input, and output vectors of $\hat{\Sigma}$ are greater than or equal to those of $\Sigma$; i.e., $\hat{n} \geq n, \hat{p} \geq p$, and $\hat{q} \geq q$. It is also assumed that $E_{0} \in \mathbf{R}^{n \times n}, A_{0} \in \mathbf{R}^{n \times n}$, $B_{0} \in \mathbf{R}^{n \times p}, C_{0} \in \mathbf{R}^{q \times n}, \hat{E}_{0} \in \mathbf{R}^{\hat{n} \times \hat{n}}, \hat{A}_{0} \in \mathbf{R}^{\hat{n} \times \hat{n}}, \hat{B}_{0} \in$ $\mathbf{R}^{\hat{n} \times \hat{p}}$, and $\hat{C}_{0} \in \mathbf{R}^{\hat{q} \times \hat{n}}$ are constant matrices and $A(\cdot)$ : $[-\tau, 0] \rightarrow \mathbf{R}^{n \times n}, B(\cdot):[-\tau, 0] \rightarrow \mathbf{R}^{n \times p}, C(\cdot):[-\tau, 0] \rightarrow$ $\mathbf{R}^{q \times n}, \hat{A}(\cdot):[-\tau, 0] \rightarrow \mathbf{R}^{\hat{n} \times \hat{n}}, \hat{B}(\cdot):[-\tau, 0] \rightarrow \mathbf{R}^{\hat{n} \times \hat{p}}$, and $\hat{C}(\cdot):[-\tau, 0] \rightarrow \mathbf{R}^{\hat{q} \times \hat{n}}$ are bounded matrix functions. It is further assumed that $\operatorname{rank}\left(E_{0}\right)=n_{e}<n$ and $\operatorname{rank}\left(\hat{E}_{0}\right)=$ $\hat{n}_{e}<\hat{n}$, which make both systems $\Sigma$ and $\hat{\Sigma}$ descriptor-type [17]. The following two assumptions are also made in order to guarantee the existence and uniqueness of solutions:

Assumption 1: $\operatorname{rank}\left(\mathcal{U} A_{0} \mathcal{V}\right)=n-n_{e}$, where the rows of $\mathcal{U}$ (respectively, columns of $\mathcal{V}$ ) span the left (respectively, right) null space of $E_{0}$.

Assumption 2: $\operatorname{rank}\left(\hat{\mathcal{U}} \hat{A}_{0} \hat{\mathcal{V}}\right)=\hat{n}-\hat{n}_{e}$, where the rows of $\hat{\mathcal{U}}$ (respectively, columns of $\hat{\mathcal{V}}$ ) span the left (respectively, right) null space of $\hat{E}_{0}$.

Under suitable initial conditions, Assumptions 1 and 2 guarantee the existence and uniqueness of solutions to (1) and (3), respectively [16]. The initial conditions for $\Sigma$ and $\hat{\Sigma}$ are assumed to be given as:

$$
x(\theta)=\phi(\theta) \quad \text { and } \quad \hat{x}(\theta)=\hat{\phi}(\theta), \quad \theta \in[-\tau, 0],
$$

respectively, for some well-defined functions $\phi:[-\tau, 0] \rightarrow$ $\mathbf{R}^{n}$ and $\hat{\phi}:[-\tau, 0] \rightarrow \mathbf{R}^{\hat{n}}$.

As also noted in [34], when Assumptions 1 and 2 hold, by a suitable state transformation, the delay-free parts of (1) and (3) can be decoupled into their differential and algebraic parts [17] (there will still be coupling through delayed dynamics, in general, however). That is, by using a state trasformation, (1)-(4) can be put into a form where

$$
E_{0}=\operatorname{bdiag}\left(I_{n_{e}}, 0\right) \text { and } A_{0}=\operatorname{bdiag}\left(A_{0}^{1}, I_{n-n_{e}}\right)
$$

and

$$
\hat{E}_{0}=\operatorname{bdiag}\left(I_{\hat{n}_{e}}, 0\right) \quad \text { and } \quad \hat{A}_{0}=\operatorname{bdiag}\left(\hat{A}_{0}^{1}, I_{\hat{n}-\hat{n}_{e}}\right)
$$

Although, in such a form, the equations may look appealing, the states may lose their physical meaning. Furthermore, some matrices/matrix functions may become ill-conditioned. Therefore, except in the only if part of Theorem 1 below, we assume that (6) and (7) may not hold in general.

The principle of extension was defined in [33] as follows: Definition 1: $\hat{\Sigma}$ is said to be an extension of $\Sigma$ if there exist full-rank matrices

$$
T \in \mathbf{R}^{\hat{n} \times n}, \quad R \in \mathbf{R}^{p \times \hat{p}}, \quad \text { and } \quad S \in \mathbf{R}^{\hat{q} \times q}
$$

such that for all $\phi(\cdot)$ and for all $\hat{u}(\cdot)$, the choice

$$
\hat{\phi}(\theta)=T \phi(\theta), \quad \theta \in[-\tau, 0]
$$

and

$$
u(t)=R \hat{u}(t), \quad t \geq-\tau
$$

implies

$$
\hat{x}(t)=T x(t), \quad t \geq-\tau
$$

and

$$
\hat{y}(t)=S y(t), \quad t \geq 0
$$

As also mentioned in [34], extension defined above is a generalization of extension, which was first defined for finitedimensional systems in [32]. In the case of finite-dimensional systems, the advantage of using extension rather than the more general principle of inclusion is that, if extension is used then any controller designed for the expanded system can be contracted for implemetation on the original system [35]. As it was shown in [34] (see Corollary 2 below), the same is also true in the present case.

The necessary and sufficient conditions for $\hat{\Sigma}$ to be an extension of $\Sigma$ are given by the following theorem, which was first presented in [33].

Theorem 1: $\hat{\Sigma}$ is an extension of $\Sigma$ if there exist full-rank matrices as in (8) such that

$$
\hat{E}_{0} T=T E_{0},
$$

$$
\begin{aligned}
& \hat{A}_{0} T=T A_{0} \text { and } \hat{A}(\theta) T=T A(\theta), \forall \theta \in[-\tau, 0], \\
& \hat{B}_{0}=T B_{0} R \text { and } \hat{B}(\theta)=T B(\theta) R, \forall \theta \in[-\tau, 0],
\end{aligned}
$$

and

$$
\hat{C}_{0} T=S C_{0} \text { and } \hat{C}(\theta) T=S C(\theta), \forall \theta \in[-\tau, 0] .
$$


Furthermore, the above conditions are also necessary if (1)(4) are written in a form such that (6) and (7) hold and that $\left[\begin{array}{ccc}B_{0}^{2} & A^{2}(\cdot) & B^{2}(\cdot)\end{array}\right]$ has full row-rank, where $B_{0}^{2}, A^{2}(\cdot)$, and $B^{2}(\cdot)$ denote the last $n-n_{e}$ rows of $B_{0}, A(\cdot)$, and $B(\cdot)$, respectively.

\section{Proof: See [33].}

As it was also indicated in [33] and [34], without any loss of generality, the matrices and the matrix functions of $\Sigma$ and $\hat{\Sigma}$ can be related as follows:

$$
\begin{gathered}
\hat{E}_{0}=T E_{0} \tilde{T}+M_{E}^{0}, \quad \hat{A}_{0}=T A_{0} \tilde{T}+M_{A}^{0}, \\
\hat{B}_{0}=T B_{0} R+M_{B}^{0}, \quad \hat{C}_{0}=S C_{0} \tilde{T}+M_{C}^{0} \\
\hat{A}(\theta)=T A(\theta) \tilde{T}+M_{A}(\theta), \quad \theta \in[-\tau, 0], \\
\hat{B}(\theta)=T B(\theta) R+M_{B}(\theta), \quad \theta \in[-\tau, 0],
\end{gathered}
$$

and

$$
\hat{C}(\theta)=S C(\theta) \tilde{T}+M_{C}(\theta), \quad \theta \in[-\tau, 0],
$$

where $T, R$, and $S$ are the matrices introduced in (8) and $\tilde{T} \in \mathbf{R}^{n \times \hat{n}}$ is a left-inverse of $T$, satisfying $\tilde{T} T=$ $I_{n}$ (such $\tilde{T}$ exists, since $T$ is of full-rank and $\hat{n} \geq n$ ). Furthermore, $M_{E}^{0} \in \mathbf{R}^{\hat{n} \times \hat{n}}, M_{A}^{0} \in \mathbf{R}^{\hat{n} \times \hat{n}}, M_{B}^{0} \in \mathbf{R}^{\hat{n} \times \hat{p}}$, and $M_{C}^{0} \in \mathbf{R}^{\hat{q} \times \hat{n}}$ are the so-called complementary matrices and $M_{A}(\cdot):[-\tau, 0] \rightarrow \mathbf{R}^{\hat{n} \times \hat{n}}, M_{B}(\cdot):[-\tau, 0] \rightarrow \mathbf{R}^{\hat{n} \times \hat{p}}$, and $M_{C}(\cdot):[-\tau, 0] \rightarrow \mathbf{R}^{\hat{q} \times \hat{n}}$ are the so-called complementary matrix functions, which are bounded matrix functions. As we will see in Section 4, this representation facilitates defining an expansion of an overlappingly decomposed system. The necessary and sufficient conditions for $\hat{\Sigma}$ to be an extension of $\Sigma$ can equivalently be stated in terms of these complementary matrices and matrix functions [33]:

Corollary 1: $\hat{\Sigma}$ is an extension of $\Sigma$ if

$$
\begin{gathered}
M_{E}^{0} T=0, \\
M_{A}^{0} T=0 \text { and } M_{A}(\theta) T=0, \forall \theta \in[-\tau, 0], \\
M_{B}^{0}=0 \text { and } M_{B}(\theta)=0, \forall \theta \in[-\tau, 0],
\end{gathered}
$$

and

$$
M_{C}^{0} T=0 \quad \text { and } \quad M_{C}(\theta) T=0, \forall \theta \in[-\tau, 0] .
$$

Furthermore, the above conditions are also necessary if (1)(4) are written in a form such that (6) and (7) hold and that $\left[\begin{array}{lll}B_{0}^{2} & A^{2}(\cdot) & B^{2}(\cdot)\end{array}\right]$ has full row-rank.

Proof: See [33].

\section{Contractibility of Controllers}

As it will be shown in the next section, in the approach of overlapping decompositions, a system is first overlappingly decomposed and then expanded so that overlapping parts appear as disjoint. A controller is then designed for this expanded system and then contracted for implementation on the original system. In order for the contracted controller work on the original system, however, it must be contractible. Contractibility of controllers for descriptor-type systems with distributed time-delay was first considered in [34]. Therefore, in this section we will summarize the results of [34].

As mentioned in [34], a possibly descriptor-type LTI timedelay system can be stabilized by a possibly descriptor-type LTI (de)centralized time-delay controller if and only if it can be stabilized by a LTI (de)centralized finite-dimensional controller (see [36] for the retarded centralized case and [37] for the most general case). Therefore, as in [34], in here, we will consider only finite-dimensional controllers. Thus, for $\Sigma$, we consider a controller, to be denoted by $\Gamma$, of the form

$$
\begin{aligned}
& \dot{z}(t)=F z(t)+G w(t) \\
& v(t)=H z(t)+K w(t)
\end{aligned}
$$

and, for $\hat{\Sigma}$, we consider a controller, to be denoted by $\hat{\Gamma}$, of the form

$$
\begin{aligned}
& \dot{\hat{z}}(t)=\hat{F} \hat{z}(t)+\hat{G} \hat{w}(t) \\
& \hat{v}(t)=\hat{H} \hat{z}(t)+\hat{K} \hat{w}(t)
\end{aligned}
$$

Here, $z(t) \in \mathbf{R}^{m}$ and $\hat{z}(t) \in \mathbf{R}^{\hat{m}}$ are the state, $w(t) \in \mathbf{R}^{q}$ and $\hat{w}(t) \in \mathbf{R}^{\hat{q}}$ are the input, and $v(t) \in \mathbf{R}^{p}$ and $\hat{v}(t) \in$ $\mathbf{R}^{\hat{p}}$ are the output vectors of, respectively, $\Gamma$ and $\hat{\Gamma}$ at time $t$. Furthermore, $F \in \mathbf{R}^{m \times m}, G \in \mathbf{R}^{m \times q}, H \in \mathbf{R}^{p \times m}$, $K \in \mathbf{R}^{p \times q}, \hat{F} \in \mathbf{R}^{\hat{m} \times \hat{m}}, \hat{G} \in \mathbf{R}^{\hat{m} \times \hat{q}}, \hat{H} \in \mathbf{R}^{\hat{p} \times \hat{m}}$, and $\hat{K} \in \mathbf{R}^{\hat{p} \times \hat{q}}$ are constant matrices. The initial conditions for $\Gamma$ and $\hat{\Gamma}$ are assumed to be given as:

$$
z(0)=\zeta_{0} \quad \text { and } \quad \hat{z}(0)=\hat{\zeta}_{0},
$$

for some $\zeta_{0} \in \mathbf{R}^{m}$ and $\hat{\zeta}_{0} \in \mathbf{R}^{\hat{m}}$, respectively.

The controllers $\Gamma$ and $\hat{\Gamma}$ are to be applied to $\Sigma$ and $\hat{\Sigma}$, respectively, by letting

$$
w(t)=y(t)-r(t) \quad \text { and } \quad \hat{w}(t)=\hat{y}(t)-\hat{r}(t)
$$

and

$$
u(t)=v(t)+d(t) \quad \text { and } \quad \hat{u}(t)=\hat{v}(t)+\hat{d}(t)
$$

for $t \geq 0$, where $r(t) \in \mathbf{R}^{q}$ and $d(t) \in \mathbf{R}^{p}$ are some external inputs (possibly a reference and a disturbance, respectively) at time $t$, for $\Sigma_{c}$, where $\Sigma_{c}$ denotes the closed-loop system obtained by applying $\Gamma$ to $\Sigma$, and $\hat{r}(t) \in \mathbf{R}^{\hat{q}}$ and $\hat{d}(t) \in \mathbf{R}^{\hat{p}}$ are some external inputs at time $t$, for $\hat{\Sigma}_{c}$, where $\hat{\Sigma}_{c}$ denotes the closed-loop system obtained by applying $\hat{\Gamma}$ to $\hat{\Sigma}$.

As it was noted in [34], although the maximum time-delay in the closed-loop systems will be $2 \tau$, due to the assumption that the loops are closed at time $t=0$, only $x(t)$ for $t \geq-\tau$ will affect $\Gamma$ and only $z(t)$ for $t \geq 0$ will affect $\Sigma$ (similarly 
for $\hat{\Gamma}$ and $\hat{\Sigma}$ ). Thus, (5) and (30) will also define the initial conditions of the closed-loop systems.

We can now present the definition of contractibility, which was first presented in [34]:

Definition 2: Suppose that the connection in (31) is made but the connection in (32) is not made. The controller $\hat{\Gamma}$ for $\hat{\Sigma}$ is said to be contractible to the controller $\Gamma$ for $\Sigma$ if there exist full-rank matrices as in (8) and a full row-rank matrix $P \in \mathbf{R}^{m \times \hat{m}}$ such that for all initial conditions $\phi(\cdot)$ of $\Sigma$, for all inputs $\hat{u}(\cdot)$ of $\hat{\Sigma}$, for all external inputs $r(\cdot)$ of $\Sigma_{c}$, and for all initial conditions $\hat{\zeta}_{0}$ of $\hat{\Gamma}$, the choice (9), (10),

$$
\zeta_{0}=P \hat{\zeta}_{0}
$$

and

$$
\hat{r}(t)=S r(t), \quad t \geq 0
$$

implies

$$
z(t)=P \hat{z}(t), \quad t \geq 0
$$

and

$$
v(t)=R \hat{v}(t), \quad t \geq 0 .
$$

Note that, the existence of a full row-rank matrix $P \in$ $\mathbf{R}^{m \times \hat{m}}$, in particular requires $\hat{m} \geq m$. However, as also indicated in [34], this is natural, since $\Sigma$, in general, forms a part of $\hat{\Sigma}$, and hence, should not require a controller with a larger dimensional state vector [8]. Contractibility is needed so that the condition (10) is satisfied after the application of the controllers. Although, however, (10) is required for $t \geq-\tau$, (36) is required only for $t \geq 0$, since the controllers are to be applied starting at time $t=0$.

The conditions for $\hat{\Gamma}$ to be contractible to $\Gamma$ are given by the following theorem, which was first presented in [34]:

Theorem 2: Suppose that $\hat{\Sigma}$ is an extension of $\Sigma$. Then, the controller $\hat{\Gamma}$ for $\hat{\Sigma}$ is contractible to the controller $\Gamma$ for $\Sigma$ if there exists a full row-rank matrix $P \in \mathbf{R}^{m \times \hat{m}}$ such that

$$
\begin{gathered}
F P=P \hat{F}, \quad G=P \hat{G} S, \\
H P=R \hat{H}, \quad \text { and } K=R \hat{K} S,
\end{gathered}
$$

where $R$ and $S$ are as in (8).

Proof: See [34].

Since a controller $\hat{\Gamma}$ is first to be designed for $\hat{\Sigma}$ and then to be contrated for implementation on $\Sigma$, it is important that any controller $\hat{\Gamma}$ for $\hat{\Sigma}$ to be contractible to a controller $\Gamma$ for $\Sigma$. As it was shown in [34], this is in fact the case if $\hat{\Sigma}$ is an extension of $\Sigma$ :

Corollary 2: If $\hat{\Sigma}$ is an extension of $\Sigma$, then any controller $\hat{\Gamma}$ of the form (28)-(29) for $\hat{\Sigma}$ is contractible to a controller $\Gamma$ of the form (26)-(27) for $\Sigma$ with

$$
\begin{gathered}
F=\hat{F}, \quad G=\hat{G} S, \\
H=R \hat{H}, \quad \text { and } \quad K=R \hat{K} S,
\end{gathered}
$$

where $R$ and $S$ are as in (8).

Proof: See [34].
Now, suppose that $\Gamma$ is applied to $\Sigma$ and $\hat{\Gamma}$ is applied to $\hat{\Sigma}$ by making the connections in (31) and (32) starting at time $t=0$. Let us denote the closed-loop system obtained by applying $\Gamma$ to $\Sigma$ by $\Sigma_{c}$ and the closed-loop system obtained by applying $\hat{\Gamma}$ to $\hat{\Sigma}$ by $\hat{\Sigma}_{c}$. In [8], for the case of retarded distributed-time-delay systems, it was proved that when the expanded system is an extension of the original system and the controller for the expanded system is contractible to the controller for the original system, then certain stability and performance relations between the two closed-loop systems hold. For stability, we can use the usual definition of asymptotic or exponential stability for timedelay systems (e.g., see [38]). For performance, a specific tracking requirement was used in [8], which is defined as follows:

Definition 3: A closed-loop system, such as $\Sigma_{c}$, is said to achieve good tracking for references $r(\cdot)$, with respect to a tolerance function $g: \mathbf{R}_{+} \rightarrow \mathbf{R}_{+}$and a disturbance bound $f:[-\tau, \infty) \rightarrow \mathbf{R}_{+}$, if, assuming that the initial state is zero, the output, $y(\cdot)$, satisfies $\|y(t)-r(t)\| \leq g(t), \forall t \geq 0$, for all disturbances which satisfy $\|d(t)\| \leq f(t), \forall t \geq-\tau$.

We note that the solution to the robust tracking controller design problem for descriptor-type time-delay systems was presented in [39].

The following theorems, the proofs of which follow the same lines as the proofs of the corresponding theorems in [8], were first presented in [34]:

Theorem 3: Suppose that $\hat{\Sigma}$ is an extension of $\Sigma, \hat{\Gamma}$ is contractible to $\Gamma$, and $\hat{\Gamma}$ stabilizes $\hat{\Sigma}$. Then, $\Gamma$ stabilizes $\Sigma$.

Theorem 4: Suppose that $\hat{\Sigma}$ is an extension of $\Sigma$ and $\hat{\Gamma}$ is contractible to $\Gamma$. Let $R$ and $S$ be as in Definition 1 and $\tilde{R}$ and $\tilde{S}$ be such that $R \tilde{R}=I_{p}$ and $\tilde{S} S=I_{q} \cdot{ }^{1}$ Suppose that $\hat{\Sigma}_{c}$ achieves good tracking for references $\hat{r}(t)=\operatorname{Sr}(t)$, $t \geq 0$, for some $r(\cdot)$, with respect to $\hat{g}: \mathbf{R}_{+} \rightarrow \mathbf{R}_{+}$and $\hat{f}:[-\tau, \infty) \rightarrow \mathbf{R}_{+}$. Then, $\Sigma_{c}$ achieves good tracking for references $r(\cdot)$, with respect to $g(\cdot):=\|\tilde{S}\| \hat{g}(\cdot)$ and $f(\cdot):=$ $\frac{1}{\|\tilde{R}\|} \hat{f}(\cdot)$.

\section{Overlapping Decompositions and Controller Design}

The results of the previous section show that, when $\hat{\Sigma}$ is an extension of $\Sigma$, a controller can first be designed for the expanded system to achieve stability and desired performance. This controller can then be contracted and implemented on the original system. The original closed-loop system will then be stable and will have the desired performance. This approach is particularly useful when the original system has an overlapping structure. In such a case overlapping decompositions can be used to obtain the expanded system which is

\footnotetext{
${ }^{1}$ There exist such $\tilde{R}$ and $\tilde{S}$ since $R$ and $S$ are of full-rank and $\hat{p} \geq$ $p$ and $\hat{q} \geq q$. Note that it makes sense to choose these matrices as the Moore-Penrose inverses [40] of $R$ and $S$, respectively, so that they will have minimum norm, which will, in turn, allow minimum tolerance function $g$ and maximum disturbance bound $f$.
} 
an extension of the original system. Decentralized controllers can then be designed for this expanded system. It would be easier to design these controllers for the expanded system, rather than directly designing a controller for the original system, since the subsystems of the expanded system would appear as disjoint. The decentralized controllers designed for the expanded system can then be contracted and implemented on the original system.

Large-scale systems may have subsystems which may overlap in many different ways [41]. For simplicity of presentation, here we will consider the case of two overlapping subsystems. In this case, the state, the input, and the output vectors of the system $\Sigma$ can be decomposed as:

$$
x=\left[\begin{array}{l}
x_{1} \\
x_{c} \\
x_{2}
\end{array}\right], \quad u=\left[\begin{array}{l}
u_{1} \\
u_{c} \\
u_{2}
\end{array}\right], \quad y=\left[\begin{array}{l}
y_{1} \\
y_{c} \\
y_{2}
\end{array}\right],
$$

where, for $i=1,2, x_{i} \in \mathbf{R}^{n_{i}}, u_{i} \in \mathbf{R}^{p_{i}}$, and $y_{i} \in \mathbf{R}^{q_{i}}$ are, respectively, the state, the input, and the output vectors of the $i^{\text {th }}$ subsystem only, and $x_{c} \in \mathbf{R}^{n_{c}}, u_{c} \in \mathbf{R}^{p_{c}}$, and $y_{c} \in \mathbf{R}^{q_{c}}$ are, respectively, the state, the input, and the output vectors of the overlapping part. Let us also partition the matrices and the matrix functions in (1)-(2) compatibly:

$$
\begin{gathered}
E_{0}=\left[\begin{array}{ccc}
E_{0}^{11} & E_{0}^{1 c} & E_{0}^{12} \\
E_{0}^{c 1} & E_{0}^{c c} & E_{0}^{c 2} \\
E_{0}^{21} & E_{0}^{2 c} & E_{0}^{22}
\end{array}\right], \\
A_{0}=\left[\begin{array}{ccc}
A_{0}^{11} & A_{0}^{1 c} & A_{0}^{12} \\
A_{0}^{c 1} & A_{0}^{c c} & A_{0}^{c 2} \\
A_{0}^{21} & A_{0}^{2 c} & A_{0}^{22}
\end{array}\right], \\
B_{0}=\left[\begin{array}{lll}
B_{0}^{11} & B_{0}^{1 c} & B_{0}^{12} \\
B_{0}^{c 1} & B_{0}^{c c} & B_{0}^{c 2} \\
B_{0}^{21} & B_{0}^{2 c} & B_{0}^{22}
\end{array}\right], \\
C_{0}=\left[\begin{array}{lll}
C_{0}^{11} & C_{0}^{1 c} & C_{0}^{12} \\
C_{0}^{c 1} & C_{0}^{c c} & C_{0}^{c 2} \\
C_{0}^{21} & C_{0}^{2 c} & C_{0}^{22}
\end{array}\right], \\
A(\cdot)=\left[\begin{array}{lll}
A^{11}(\cdot) & A^{1 c}(\cdot) & A^{12}(\cdot) \\
A^{c 1}(\cdot) & A^{c c}(\cdot) & A^{c 2}(\cdot) \\
A^{21}(\cdot) & A^{2 c}(\cdot) & A^{22}(\cdot)
\end{array}\right], \\
B(\cdot)=\left[\begin{array}{lll}
B^{11}(\cdot) & B^{1 c}(\cdot) & B^{12}(\cdot) \\
B^{c 1}(\cdot) & B^{c c}(\cdot) & B^{c 2}(\cdot) \\
B^{21}(\cdot) & B^{2 c}(\cdot) & B^{22}(\cdot)
\end{array}\right],
\end{gathered}
$$

and

$$
C(\cdot)=\left[\begin{array}{lll}
C^{11}(\cdot) & C^{1 c}(\cdot) & C^{12}(\cdot) \\
C^{c 1}(\cdot) & C^{c c}(\cdot) & C^{c 2}(\cdot) \\
C^{21}(\cdot) & C^{2 c}(\cdot) & C^{22}(\cdot)
\end{array}\right]
$$

Then, to obtain an extension of $\Sigma$, the matrices in (8) can be chosen as

$$
T=\left[\begin{array}{ccc}
I_{n_{1}} & 0 & 0 \\
0 & I_{n_{c}} & 0 \\
0 & I_{n_{c}} & 0 \\
0 & 0 & I_{n_{2}}
\end{array}\right]
$$

$$
R=\left[\begin{array}{cccc}
I_{p_{1}} & 0 & 0 & 0 \\
0 & I_{p_{c}} & I_{p_{c}} & 0 \\
0 & 0 & 0 & I_{p_{2}}
\end{array}\right]
$$

and

$$
S=\left[\begin{array}{ccc}
I_{q_{1}} & 0 & 0 \\
0 & I_{q_{c}} & 0 \\
0 & I_{q_{c}} & 0 \\
0 & 0 & I_{q_{2}}
\end{array}\right]
$$

Furthermore, the matrix $\tilde{T}$ can be chosen as the MoorePenrose inverse [40] of $T$ :

$$
\tilde{T}=\left[\begin{array}{cccc}
I_{n_{1}} & 0 & 0 & 0 \\
0 & \frac{1}{2} I_{n_{c}} & \frac{1}{2} I_{n_{c}} & 0 \\
0 & 0 & 0 & I_{n_{2}}
\end{array}\right]
$$

Then, an extension $\hat{\Sigma}$ of $\Sigma$ can be obtained as described by (3)-(4), where the matrices and the matrix functions in (3)-(4) are chosen as in (17)-(21), with the following complementary matrices and matrix functions:

$$
\begin{aligned}
& M_{E}^{0}=\left[\begin{array}{cccc}
0 & \frac{1}{2} E_{0}^{1 c} & -\frac{1}{2} E_{0}^{1 c} & 0 \\
0 & \frac{1}{2} E_{0}^{c c} & -\frac{1}{2} E_{0}^{c c} & 0 \\
0 & -\frac{1}{2} E_{0}^{c c} & \frac{1}{2} E_{0}^{c c} & 0 \\
0 & -\frac{1}{2} E_{0}^{2 c} & \frac{1}{2} E_{0}^{2 c} & 0
\end{array}\right] \\
& M_{A}^{0}=\left[\begin{array}{cccc}
0 & \frac{1}{2} A_{0}^{1 c} & -\frac{1}{2} A_{0}^{1 c} & 0 \\
0 & \frac{1}{2} A_{0}^{c c} & -\frac{1}{2} A_{0}^{c c} & 0 \\
0 & -\frac{1}{2} A_{0}^{c c} & \frac{1}{2} A_{0}^{c c} & 0 \\
0 & -\frac{1}{2} A_{0}^{2 c} & \frac{1}{2} A_{0}^{2 c} & 0
\end{array}\right] \text {, } \\
& M_{B}^{0}=0, \\
& M_{C}^{0}=\left[\begin{array}{cccc}
0 & \frac{1}{2} C_{0}^{1 c} & -\frac{1}{2} C_{0}^{1 c} & 0 \\
0 & \frac{1}{2} C_{0}^{c c} & -\frac{1}{2} C_{0}^{c c} & 0 \\
0 & -\frac{1}{2} C_{0}^{c c} & \frac{1}{2} C_{0}^{c c} & 0 \\
0 & -\frac{1}{2} C_{0}^{2 c} & \frac{1}{2} C_{0}^{2 c} & 0
\end{array}\right] \\
& M_{A}(\cdot)=\left[\begin{array}{cccc}
0 & \frac{1}{2} A^{1 c}(\cdot) & -\frac{1}{2} A^{1 c}(\cdot) & 0 \\
0 & \frac{1}{2} A^{c c}(\cdot) & -\frac{1}{2} A^{c c}(\cdot) & 0 \\
0 & -\frac{1}{2} A^{c c}(\cdot) & \frac{1}{2} A^{c c}(\cdot) & 0 \\
0 & -\frac{1}{2} A^{2 c}(\cdot) & \frac{1}{2} A^{2 c}(\cdot) & 0
\end{array}\right] \text {, } \\
& M_{B}(\cdot)=0 \text {, and } \\
& M_{C}(\cdot)=\left[\begin{array}{cccc}
0 & \frac{1}{2} C^{1 c}(\cdot) & -\frac{1}{2} C^{1 c}(\cdot) & 0 \\
0 & \frac{1}{2} C^{c c}(\cdot) & -\frac{1}{2} C^{c c}(\cdot) & 0 \\
0 & -\frac{1}{2} C^{c c}(\cdot) & \frac{1}{2} C^{c c}(\cdot) & 0 \\
0 & -\frac{1}{2} C^{2 c}(\cdot) & \frac{1}{2} C^{2 c}(\cdot) & 0
\end{array}\right] .
\end{aligned}
$$

These matrices and matrix functions are chosen to minimize the interactions between the expanded subsystems among all complementary matrices and matrix functions which satisfy (22)-(25). Since (22)-(25) are satisfied, by Corollary 1, $\hat{\Sigma}$ is an extension of $\Sigma$.

We can then decompose the state, the input, and the output vectors of $\hat{\Sigma}$ as:

$$
\hat{x}=\left[\begin{array}{l}
\hat{x}_{1} \\
\hat{x}_{2}
\end{array}\right], \hat{u}=\left[\begin{array}{l}
\hat{u}_{1} \\
\hat{u}_{2}
\end{array}\right], \hat{y}=\left[\begin{array}{l}
\hat{y}_{1} \\
\hat{y}_{2}
\end{array}\right],
$$

where, for $i=1,2, \hat{x}_{i} \in \mathbf{R}^{\hat{n}_{i}}, \hat{n}_{i}:=n_{i}+n_{c}, \hat{u}_{i} \in \mathbf{R}^{\hat{p}_{i}}, \hat{p}_{i}:=$ $p_{i}+p_{c}$, and $\hat{y}_{i} \in \mathbf{R}^{\hat{q}_{i}}, \hat{q}_{i}:=q_{i}+q_{c}$. Then, $\hat{\Sigma}$ is composed of 
two subsystems: $\hat{\Sigma}_{1}$ and $\hat{\Sigma}_{2}$, where $\hat{x}_{i}, \hat{u}_{i}$, and $\hat{y}_{i}$ respectively form the state, the input, and the output vectors of $\hat{\Sigma}_{i}$, for $i=1,2$. Then, for each $\hat{\Sigma}_{i}(i=1,2)$, a local controller $\hat{\Gamma}_{i}$ of the form

$$
\begin{aligned}
& \dot{\hat{z}}_{i}(t)=\hat{F}_{i} \hat{z}_{i}(t)+\hat{G}_{i} \hat{w}_{i}(t) \\
& \hat{v}_{i}(t)=\hat{H}_{i} \hat{z}_{i}(t)+\hat{K}_{i} \hat{w}_{i}(t)
\end{aligned}
$$

can be designed, e.g., by the method of [42] or [43]. This controller is to be applied to $\hat{\Sigma}_{i}$ by letting

$$
\hat{w}_{i}(t)=\hat{y}_{i}(t)-\hat{r}_{i}(t) \quad \text { and } \quad \hat{u}_{i}(t)=\hat{v}_{i}(t)+\hat{d}_{i}(t)
$$

for $t \geq 0$, where $\hat{r}_{i}(t) \in \mathbf{R}^{\hat{q}_{i}}$ and $\hat{d}_{i}(t) \in \mathbf{R}^{\hat{p}_{i}}$ are some external inputs at time $t$ for the $i^{\text {th }}$ local closed-loop system.

For $i=1,2$, let us make the following decompositions:

$$
\hat{G}_{i}=\left[\begin{array}{ll}
G_{i}^{1} & G_{i}^{2}
\end{array}\right], \quad \hat{H}_{i}=\left[\begin{array}{c}
H_{i}^{1} \\
H_{i}^{2}
\end{array}\right],
$$

and

$$
\hat{K}_{i}=\left[\begin{array}{cc}
K_{i}^{11} & K_{i}^{12} \\
K_{i}^{21} & K_{i}^{22}
\end{array}\right]
$$

where $G_{1}^{1}$ has $q_{1}$ columns, $G_{2}^{2}$ has $q_{2}$ columns, $H_{1}^{1}$ has $p_{1}$ rows, $H_{2}^{2}$ has $p_{2}$ rows, $K_{1}^{11}$ has $p_{1}$ rows and $q_{1}$ columns, and $K_{2}^{22}$ has $p_{2}$ rows and $q_{2}$ columns.

A decentralized controller $\hat{\Gamma}$ for $\hat{\Sigma}$ can then be obtained as in (28)-(29), where

$$
\begin{gathered}
\hat{F}=\operatorname{bdiag}\left(\hat{F}_{1}, \hat{F}_{2}\right), \quad \hat{G}=\operatorname{bdiag}\left(\hat{G}_{1}, \hat{G}_{2}\right), \\
\hat{H}=\operatorname{bdiag}\left(\hat{H}_{1}, \hat{H}_{2}\right),
\end{gathered}
$$

and

$$
\hat{K}=\operatorname{bdiag}\left(\hat{K}_{1}, \hat{K}_{2}\right)
$$

This controller can then be contracted to a controller $\Gamma$, described by (26)-(27), where the matrices are obtained using (39)-(40) as follows:

$$
\begin{gathered}
F=\operatorname{bdiag}\left(\hat{F}_{1}, \hat{F}_{2}\right), \\
G=\left[\begin{array}{ccc}
G_{1}^{1} & G_{1}^{2} & 0 \\
0 & G_{2}^{1} & G_{2}^{2}
\end{array}\right], \quad H=\left[\begin{array}{cc}
H_{1}^{1} & 0 \\
H_{1}^{2} & H_{2}^{1} \\
0 & H_{2}^{2}
\end{array}\right],
\end{gathered}
$$

and

$$
K=\left[\begin{array}{ccc}
K_{1}^{11} & K_{1}^{12} & 0 \\
K_{1}^{21} & K_{1}^{22}+K_{2}^{11} & K_{2}^{12} \\
0 & K_{2}^{21} & K_{2}^{22}
\end{array}\right]
$$

The overlapping decentralized structure of the controller is evident from these matrices. Assuming that $\hat{\Gamma}$ stabilizes $\hat{\Sigma}$, by Theorem 3, the contracted controller $\Gamma$ stabilizes the original system $\Sigma$. Furthermore, if $\hat{\Sigma}_{c}$ achieves good tracking for references $\hat{r}(t)=S r(t), t \geq 0$, for some $r(\cdot)$, with respect to $\hat{g}: \mathbf{R}_{+} \rightarrow \mathbf{R}_{+}$and $\hat{f}:[-\tau, \infty) \rightarrow \mathbf{R}_{+}$, then, by Theorem $4, \Sigma_{c}$ achieves good tracking for references $r(\cdot)$, with respect to $g(\cdot)=\hat{g}(\cdot)$ and $f(\cdot)=\hat{f}(\cdot)$. $^{2}$

${ }^{2}$ As $\tilde{R}$ and $\tilde{S}$, we use the Moore-Penrose inverses of $R$ and $S$ in (43) and (44) so that $\|\tilde{R}\|=\|\tilde{S}\|=1$.

\section{Conclusions}

Decentralized controller design using overlapping decompositions has been considered for descriptor-type systems with distributed time-delay. First, the principle of extension and contractibility of controllers have been presented, as they were first introduced in [33] and [34], respectively. Then, controller design using overlapping decompositions has been presented. Although the case of only two overlapping subsystems has been presented for simplicity, the results can be extended to systems which may have more subsystems which may overlap in different ways. Furthermore, although only finite-dimensional controllers has been considered, the results can be generalized to time-delay controllers. ${ }^{3}$

In the proposed approach, a given large-scale system is first overlappingly decomposed into a number of subsystems. This system is then expanded such that the expanded system is an extension of the original system and the subsystems of the expanded system appear as disjoint. Decentralized controllers are then designed for this expanded system. It would be easier to design these controllers for the expanded system, rather than directly designing a controller for the original system, since the subsystems of the expanded system would appear as disjoint. Any decentralized controller design method, such as [42] or [43], devised for descriptortype time-delay systems can be employed to design these controllers. The decentralized controllers designed for the expanded system are contracted and implemented on the original system in the final phase. As it was shown in Section 4, the controllers designed by this approach would have an overlapping decentralized structure, which may be desirable in many applications. Furthermore, by Theorems 3 and 4, the stability and desired performance for the original system would be guaranteed, if the controllers for the expanded system are designed to stabilize and achieve desired performance.

\section{Acknowledgement}

This work has been supported by the Scientific Research Projects Commission of Eskişehir Technical University under grant number 20ADP235.

\section{References}

[1] S.-I. Niculescu, Delay Effects on Stability: A Robust Control Approach, Lecture Notes in Control and Information Sciences, No. 269. London: Springer-Verlag, 2001

[2] L. Bakule, "Decentralized control: An overview," Annual Reviews in Control, vol. 32, pp. 87-98, 2008

[3] J.-P. Richard, "Time-delay systems: an overview of some recent advances and open problems," Automatica, vol. 39, pp. 1667-1694, 2003

[4] K. L. Cooke and Z. Grossman, "Discrete delay, distributed delay and stability switches," Journal of Mathematical Analysis and Applications, vol. 86, pp. 592-627, 1982.

${ }^{3}$ Even though it is stated in Section 4 that finite-dimensional controllers are sufficient for stabilization (assuming that the given system is stabilizable), time-delay controllers may have certain advantages [44]. 
[5] L. Berezansky and E. Braverman, "Oscillation properties of a logistic equation with distributed delay," Nonlinear Analysis: Real World Applications, vol. 4, pp. 1-19, 2003.

[6] W. Michiels, C.-I. Morărescu, and S.-I. Niculescu, "Consensus problems with distributed delays, with application to traffic flow models," SIAM Journal on Control and Optimization, vol. 48, pp. 77-101, 2009.

[7] R. Sipahi, F. M. Atay, and S.-I. Niculescu, "Stability of traffic flow behaviour with distributed delays modeling the memory effects of the drivers," SIAM Journal on Applied Mathematics, vol. 68, pp. 738-759, 2008.

[8] A. İftar, "Extension principle and controller design for systems with distributed time-delay," Kybernetika, vol. 53, pp. 630-652, 2017.

[9] L. Xie, E. Fridman, and U. Shaked, "Robust $\mathcal{H}_{\infty}$ control of distributed delay systems with application to combustion control," IEEE Transactions on Automatic Control, vol. 46, pp. 1930-1935, 2001.

[10] F. M. Atay and A. Hutt, "Neural fields with distributed transmission speeds and long-range feedback delays," SIAM Journal on Applied Dynamical Systems, vol. 5, pp. 670-698, 2006.

[11] B. Rahman, K. B. Blyuss, and Y. N. Kyrychko, "Dynamics of neural systems with discrete and distributed time delays," SIAM Journal on Applied Dynamical Systems, vol. 14, pp. 2069-2095, 2015.

[12] H. Özbay, C. Bonnet, and J. Clairambault, "Stability analysis of systems with distributed delays and application to hematopoietic cell maturation dynamics," in Proceedings of the IEEE Conference on Decision and Control, (Cancun, Mexico), pp. 2050-2055, Dec. 2008.

[13] V. S. H. Rao and P. R. S. Rao, "Global stability in chemostat models involving time delays and wall growth," Nonlinear Analysis: Real World Applications, vol. 5, pp. 141-158, 2004

[14] L. Ma, M. Xu, R. Jia, and H. Ye, "Exponential $H_{\infty}$ filter design for stochastic Markovian jump systems with both discrete and distributed time-varying delays," Kybernetika, vol. 50, pp. 491-511, 2014.

[15] A. İftar, "A robust controller design approach for descriptor-type systems with distributed time-delay," in Proceedings of the European Control Conference, (Rotterdam, Netherlands), pp. 429-433, June 2021.

[16] J. K. Hale and S. M. Verduyn-Lunel, Introduction to Functional Differential Equations. New York: Springer-Verlag, 1993.

[17] G.-R. Duan, Analysis and Design of Descriptor Linear Systems. New York: Springer, 2010.

[18] N. Mollet, ed., Remote and Telerobotics. InTech, 2010.

[19] D. D. Šiljak, Large-Scale Dynamic Systems: Stability and Structure. New York: North-Holland, 1978.

[20] D. D. Šiljak, Decentralized Control of Complex Systems. San Diego: Academic Press, Inc., 1991.

[21] M. Ikeda and D. D. SSiljak, "Overlapping decompositions, expansions, and contractions of dynamic systems," Large Scale Systems, vol. 1, pp. 29-38, 1980.

[22] M. Ikeda, D. D. Šiljak, and D. E. White, "Decentralized control with overlapping information sets," Journal of Optimization Theory and Applications, vol. 34, pp. 279-310, 1981.

[23] A. Iftar and F. Khorrami, "A comparison of multiple time-scale analysis and overlapping decomposition," IEEE Transactions on Systems, Man, and Cybernetics, vol. SMC-19, pp. 1296-1300, 1989.

[24] A. İftar and Ü. Özgüner, "Local LQG/LTR controller design for decentralized systems," IEEE Transactions on Automatic Control, vol. AC-32, pp. 926-930, 1987.

[25] A. İftar and Ü. Özgüner, "Overlapping decompositions, expansions, contractions, and stability of hybrid systems," IEEE Transactions on Automatic Control, vol. 43, pp. 1040-1055, 1998.

[26] Ü. Özgüner, C. Hatipoğlu, A. İftar, and K. Redmill, "Hybrid control design for a three vehicle scenario demonstration using overlapping decompositions," in Hybrid Systems IV, Lecture Notes in Computer Science, vol 1273, P. J. Antsaklis, Ed., (Springer Verlag), pp. 294 328, 1997.

[27] S. S. Stanković, M. J. Stanojević, and D. D. Šiljak, "Decentralized overlapping control of a platoon of vehicles," IEEE Transactions on Control Systems Technology, vol. 8, pp. 816-832, 2000.

[28] B. Ataşlar and A. İftar, "Decentralized routing controller design using overlapping decompositions," International Journal of Control, vol. 72, pp. 1175-1192, 1999.

[29] A. Aybar and A. İftar, "Overlapping decompositions and expansions of Petri nets," IEEE Transactions on Automatic Control, vol. 47, pp. 511515,2002

[30] A. İftar, "Decentralized robust control based on overlapping decompositions," in Preprints of the 10th IFAC Symposium on Large Scale Systems, (Osaka, Japan), pp. 605-609, July 2004.
[31] M. Ikeda, D. D. Šiljak, and D. E. White, "An inclusion principle for dynamic systems," IEEE Transactions on Automatic Control, vol. AC29, pp. 244-249, 1984

[32] A. İftar and Ü. Özgüner, "Contractible controller design and optimal control with state and input inclusion," Automatica, vol. 26, pp. 593597, 1990.

[33] A. İftar, "Extension principle for descriptor-type systems with distributed time-delay," in Proceedings of the 24th International Conference on System Theory, Control and Computing, (Sinaia, Romania), Oct. 2020 .

[34] A. İftar, "Controller design for descriptor-type systems with distributed time-delay using extension," WSEAS Transactions on Circuits and Systems, vol. 20, pp. 27-32, 2021.

[35] A. İftar, "Decentralized estimation and control with overlapping input, state, and output decomposition," Automatica, vol. 29, pp. 511-516, 1993.

[36] E. W. Kamen, P. P. Khargonekar, and A. Tannenbaum, "Stabilization of time-delay systems using finite-dimensional compensators," IEEE Transactions on Automatic Control, vol. AC-30, pp. 75-78, 1985.

[37] H. E. Erol and A. İftar, "Stabilization of decentralized descriptor-type neutral time-delay systems by time-delay controllers," Automatica, vol. 64, pp. 262-269, 2016.

[38] W. Michiels and S.-I. Niculescu, Stability, Control, and Computation for Time-Delay Systems: An Eigenvalue-Based Approach. Philadelphia: SIAM, 2014

[39] A. Ifftar, "Robust tracking and disturbance rejection for descriptor-type neutral time-delay systems," WSEAS Transactions on Systems, vol. 20, pp. 48-53, 2021.

[40] R. Penrose and J. A. Todd, "On best approximate solutions of linear matrix equations," Mathematical Proceedings of the Cambridge Philosophical Society, vol. 52, pp. 17-19, 1956.

[41] A. İftar, "Overlapping decentralized dynamic optimal control," International Journal of Control, vol. 58, pp. 187-209, 1993.

[42] H. E. Erol and A. İftar, "Decentralized controller design by continuous pole placement for neutral time-delay systems," Transactions of the Institute of Measurement and Control, vol. 39, pp. 297-311, 2017.

[43] S. M. Özer and A. İftar, "Eigenvalue optimisation-based centralised and decentralised stabilisation of time-delay systems," International Journal of Control, 2021. Published online, DOI: 10.1080/00207179.2021.1906446.

[44] H. E. Erol and A. İftar, "Decentralized time-delay controller design for systems described by delay differential-algebraic equations," Transactions of the Institute of Measurement and Control, vol. 43, pp. 31293148,2021

\section{Contribution of Individual Authors to the Creation of a Scientific Article (Ghostwriting Policy)}

The author contributed in the present research, at all stages from the formulation of the problem to the final findings and solution.

\section{Sources of Funding for Research Presented in a Scientific Article or Scientific Article Itself} No funding was received for conducting this study.

\section{Conflict of Interest}

The author has no conflict of interest to declare that is relevant to the content of this article.

\section{Creative Commons Attribution License 4.0 (Attribution 4.0 International, CC BY 4.0)}

This article is published under the terms of the Creative Commons Attribution License 4.0 https://creativecommons.org/licenses/by/4.0/deed.en _US 